\title{
Reactive biased target ion beam deposited W-DLC nanocomposite thin films - Microstructure and its mechanical properties
}

\author{
P. Vijai Bharathy ${ }^{\text {a,d,* }}$, Q. Yang ${ }^{b}$, M.S.R.N. Kiran ${ }^{\text {c }}$, JongJoo Rha ${ }^{\text {d, } * *}$, D. Nataraj ${ }^{\text {a }}$ D. Mangalaraj ${ }^{\text {e }}$ \\ a Thin films and Nanomaterials Lab, Department of Physics, Bharathiar University, Coimbatore, India \\ ${ }^{\mathrm{b}}$ Department of Mechanical Engineering, University of Saskatchewan, Saskatoon, Canada \\ c Department of Materials Engineering, Indian Institute of Science, Bangalore, India \\ d Functional coating group, Korea Institute of Material Science, Changwon, South Korea \\ e Department of Nanoscience and Technology, Bharathiar University, Coimbatore, India
}

\section{A R T I C L E I N F O}

\section{Article history:}

Received 23 August 2011

Received in revised form 21 November 2011

Accepted 20 December 2011

Available online 11 January 2012

\section{Keywords:}

Diamond like carbon

Tungsten

Reactive biased target ion beam deposition XPS

Mechanical properties

\begin{abstract}
A B S T R A C T
Tungsten incorporated diamond like carbon (W-DLC) nanocomposite thin films with variable fractions of tungsten were deposited by using reactive biased target ion beam deposition technique. The influence of tungsten incorporation on the microstructure, surface topography, mechanical and tribological properties of the DLC were studied using X-ray photoelectron spectroscopy (XPS), Raman spectroscopy, Atomic force microscope (AFM), transmission electron microscopy (TEM), nano-indentation and nano-scratch tests. The amount of $\mathrm{W}$ in films gets increases with increasing target bias voltage and most of the incorporated $\mathrm{W}$ reacts with carbon to form WC nanoclusters. Using TEM and FFT pattern, it was found that spherical shaped WC nanoclusters were uniformly dispersed in the DLC matrix and attains hexagonal $\left(\mathrm{W}_{2} \mathrm{C}\right)$ crystalline structure at higher $\mathrm{W}$ concentration. On the other hand, the incorporation of tungsten led to increase the formation of $\mathrm{C}$-sp ${ }^{2}$ hybridized bonding in DLC network and which is reflected in the hardness and elastic modulus of WDLC films. Moreover, W-DLC films show very low friction coefficient and increased adhesion to the substrate than the DLC film, which could be closely related to its unique nanostructure of the W incorporated thin films. (c) 2011 Elsevier B.V. All rights reserved.
\end{abstract}

\section{Introduction}

Recently, there has been a growing research interest for the development of novel engineering materials, especially in the form of thin protective coatings. Among other transition metal and ceramic protective coatings, diamond like carbon thin films have gained much interest owing to their excellent properties like high hardness, high wear resistance, optical transparency, low friction coefficient, chemical inertness, excellent biocompatibility and high corrosion resistance $[1,2]$. However, the preparation and application of DLC films have been restricted by its high internal compressive stress, which greatly limits the adhesive strength between the film and the substrate. Recently many researchers have adopted different methodologies to resolve this problem, for example, by doping and/or introducing interlayer between the film and the substrate. Among these methodologies, doping with metallic or non-metallic elements like Ti, Cr, W, $\mathrm{Ni}, \mathrm{Si}, \mathrm{Ag}$ and $\mathrm{Co}$ into the DLC matrix has shown promising outcome not only in reducing the internal stress, but also in enhancing the

\footnotetext{
* Correspondence to: P. Vijai Bharathy, Thin films and Nanomaterials Lab, Department of Physics, Bharathiar University, Coimbatore, India.

** Corresponding author.

E-mail addresses: pvijay126@gmail.com (P. Vijai Bharathy), jjrha@kims.re.kr (J. Rha).
}

adhesion strength [3], improving the wear resistance [4], reducing the friction coefficient [5], increasing the biocompatibility [6] and other properties of DLC thin film. Recently, it was found that this is mainly due to the presence of metal carbide clusters in the amorphous DLC host matrix. Few researchers suggested that the formation of nanosized composite clusters in the amorphous DLC matrix alters the bonding and microstructure of carbon network; hence the final properties of nanocomposite film get altered [7-9]. Recently, Corbella et al. [10] showed that the presence of metal carbide clusters increased the surface roughness of the film and influenced the mechanical properties of DLC differently, depending on the type and the concentration of incorporated metal. In an another study on MoDLC films, Ji et al. [11] found that the uniform dispersion of nanosized $\mathrm{MoC}$ crystallites in the carbon matrix, decreased the internal stress and increased the adhesion strength to a greater extend. However, Sedlackova et al. [12] and Wang et al. [13] have proposed that there is no clear dependence on the mechanical hardness of the Me-DLC films with respect to the increase in the metal concentrations and with the formation of metal carbide clusters in the carbon matrix. Also, Foong et al. [14] and Ming et al. [15] suggested that the increase in the adhesion strength of Me-DLC film is not only due to the formation of metal carbide cluster, but also due to the formation of $\mathrm{SiC}$ bonds between the substrate and the Me-DLC film, from the energetic carbon ion bombardment during thin film deposition. Hence, till 
now the role of the incorporated metallic element on the microstructure and the final properties of the DLC nanocomposite films are not yet fully understood. Overall, a careful consideration is required for the selection of suitable metal, level of metal, deposition temperature and the incorporation methodology. Moreover, the energy of the impinging carbon and metal ions on the growing surface of the DLC films has been a crucial factor in determining the microstructure and the final properties of the Me-DLC nanocomposite thin films.

Nowadays Me-DLC nanocomposite thin films have been widely prepared by various physical vapour deposition techniques which include reactive sputter deposition [16], pulsed laser deposition [17], microwave PACVD [18] and hybrid PVD/CVD plasma assisted deposition [19], all these deposition techniques are unique of its own. However, most of the depositions were carried out by applying substrate bias voltage, high energy ion bombardment and deposition at elevated temperatures. These deposition conditions may lead to easy graphitisation of carbon matrix and deterioration in the final properties of Me-DLC films. Hence in order to avoid these, we used a hybrid deposition technique known as reactive biased target ion beam deposition (RBTIBD) which is highly reliable to produce a very high density of inert gas ions with a very low energy for uniform deposition of thin films. This system combines an ion beam deposition with ion beam sputtering, and offers independent control of ion energy and plasma densities than conventional sputtering techniques. Also this deposition technique possesses a unique addition feature of controlling the metal concentration in the DLC matrix by just adjusting the target bias voltage and hence target poisoning by methane gas is completely eliminated. Most importantly, the usage of EH ion sources for the deposition of carbon based thin films has an advantage over other ion sources is by supplying low ion beam energy and high ionic current which are more suitable for altering the microstructure and improves the properties of carbon based thin films [20]. The other advantages of this technique over the conventional sputtering methods are the low overspill contamination effect and potential for scaling up for industrial coating applications.

In this work, we have used this hybrid deposition technique with low ion energy of $30 \mathrm{eV}$ with high ion current of $2 \mathrm{~A}$ to deposit highly adherable tungsten incorporated DLC nanocomposite thin films. All the depositions were carried out at room temperature and also without applying substrate biasing voltages, which was not covered in our previous report [21]. To the best of our knowledge, no previous work has been reported on the fabrication of W-DLC nanocomposite films under these conditions using RBTIBD method. Here, we focussed mainly on understanding the relationship between the chemical composition and the mechanical properties of W-DLC nanocomposite thin films because the role of incorporated tungsten element in low fractions on the microstructure and mechanical properties of the DLC film is not yet fully understood.

\section{Experimental procedure}

Tungsten incorporated diamond like carbon thin films with variable W fractions were deposited onto a p-type (100) Si substrate by using reactive biased target ion beam deposition method. The detail working mechanism of the deposition system was explained elsewhere [22]. High purity (99.9995\%) methane $\left(\mathrm{CH}_{4}\right)$ and Argon ( $\left.\mathrm{Ar}\right)$ gases were mixed in the ratio of 1:1 and introduced into end-hall ion source I to deposit pure DLC thin film. Similarly for the deposition of W-DLC nanocomposite films, the hydrocarbon ion beam with ion energy of $30 \mathrm{eV}$ with high ion current of $2 \mathrm{~A}$ was directed towards the substrate to deposit DLC film, at the same time tungsten was sputtered by using high purity Ar gas using another end-hall ion source II using the same ion energy ( $30 \mathrm{eV}$ ) and ion current (2 A). The W sputtering yield was increased by increasing the negative bias voltage from $-300 \mathrm{~V}$ to $-700 \mathrm{~V}$. During the deposition, the argon/methane gas ratio and ion beam energy were fixed constant, only the $\mathrm{W}$ target bias voltage was increased to alter the $\mathrm{W}$ sputtering yield. Also, the substrate stage was placed at an angle of $45^{\circ}$ and it was rotated about its axis at constant speed of $3 \mathrm{rpm}$ to achieve better uniformity. The deposition duration was kept as $45 \mathrm{~min}$ in order to obtain thickness of $\sim 130 \mathrm{~nm}$ for all W-DLC nanocomposite thin films. All the depositions were made at room temperature using separate cooling system to maintain the substrate stage temperature at $25^{\circ} \mathrm{C}$. The thickness of the films was measured to be around $130 \pm 2 \mathrm{~nm}$ using the surface profilometer measurements.

The chemical composition and binding energies of W-DLC thin film with different $\mathrm{W}$ concentration was analysed by X-ray photoelectron spectroscopy (XPS) on VG Microtech Multilab 3000 spectrometer. An $\mathrm{Mg} \mathrm{K \alpha}(h=1253.6 \mathrm{eV}, 1 \mathrm{eV}=1.6302 \times 10-19 \mathrm{~J}) 300$ W X-ray source was used; spectra of the W $4 \mathrm{f}$ and $\mathrm{C} 1 \mathrm{~s}$ core shells were collected with an energy resolution of $0.2 \mathrm{eV}$. The chemical compositions of the films were determined by deconvoluted the XPS spectra using XPSpeak 4.1 software. Also in order to know the chemical composition at the core of the sample, the sample surface were etched to a depth of about $20 \mathrm{~nm}$ using $\mathrm{Ar}+$ ion beam, so as to remove the oxygen contaminants. Transmission electron microscopy (TEM) and high resolution transmission electron microscopy (HRTEM) investigations were carried out by a Philips CM20 $200 \mathrm{kV}$ analytical microscope and a JEOL $3010300 \mathrm{kV}$ microscope, respectively. Fast Fourier transform (FFT) pattern was used to analysis the lattice spacing of crystalline phase in the film. The surface topography of the thin film was analysed using VEECO atomic force microscope.

The mechanical properties were measured on the DLC and W-DLC films with a nanoindenter (Triboindenter of Hysitron, Minneapolis, USA) with an in situ imaging capability. The machine continuously monitors and records the load, $P$, and displacement, $h$, of the indenter with force and displacement resolutions of $1 \mathrm{nN}$ and $0.2 \mathrm{~nm}$, respectively. A three sided pyramidal Berkovich diamond indenter with a tip radius of $\sim 100 \mathrm{~nm}$ was used to indent the nanocomposite thin film. A partial load function is used in this experiment. This type of partial load function is useful to determine hardness at each depth at a single place. On each film, 10 indents were made with the force varying from 5000 to $200 \mu \mathrm{N}$ up to a maximum penetration depth of $\sim 90 \mathrm{~nm}$. The indentation impressions were captured immediately after unloading, so as to avoid time-dependent elastic recovery of the residual impression. The $P-h$ curves were analyzed using the standard Oliver-Pharr method [23] to extract the elastic modulus, $E$, of the crystal in that orientation. However, this method was not employed for estimation of the hardness, $H$, as there was significant plastic flow during indentation, which resulted in pile-up of material against the indenter. Such pile-up, when present, makes the estimation of $H$ using the $O-P$ method inaccurate. Therefore, $H$ was determined from the maximum indentation load, $P_{\max }$, divided by the contact area, A. The latter was estimated from images of the indentation impressions. In addition to that the adhesion and friction coefficient of the nanocomposite thin films were evaluated by using nanoindentation system in scratch mode with a conospherical diamond tip of size $(10 \mu \mathrm{m})$ in a ramping load range between 0 to $1 \mathrm{mN}$ in $30 \mathrm{~s}$.

\section{Result and discussions}

\subsection{X-ray Photoelectron Spectroscopic Analysis (XPS)}

The chemical composition and bonding nature of the un-etched DLC and W-DLC thin films were studied quantitatively by using XPS. The relative atomic percentage of the components at the surface of the films was evaluated using the high resolution XPS spectra and presented in Table 1. The elemental concentration of $\mathrm{W}$ in the DLC matrix get increases from 6.1 to 13.7 at.\% with increase of target bias voltage from $-300 \mathrm{~V}$ to $-700 \mathrm{~V}$. It was clearly seen that the sputtering yield of $\mathrm{W}$ is directly proportional to the target bias voltage. In 
Table 1

Surface chemical composition of unetched DLC and W-DLC thin films prepared at different target bias voltage.

\begin{tabular}{llllr}
\hline Sample & $\begin{array}{l}\text { Target bias } \\
\text { voltage }(\mathrm{V})\end{array}$ & \multicolumn{3}{c}{ Concentration } \\
\cline { 3 - 5 } & & $\mathrm{C}$ & $\mathrm{W}$ & $\mathrm{O}$ \\
\hline DLC & 0 & 92.4 & 0 & 7.6 \\
W-DLC 1 & -300 & 65.1 & 6.1 & 28.9 \\
W-DLC 2 & -400 & 58.4 & 7.2 & 30.1 \\
W-DLC 3 & -500 & 55.2 & 8.2 & 33.1 \\
W-DLC 4 & -600 & 52.7 & 10.4 & 35.3 \\
W-DLC 5 & -700 & 49.3 & 13.7 & 36.7 \\
\hline
\end{tabular}

addition to that the atomic concentration of oxygen at the surface of the air-exposed W-DLC thin film gets increased with the increase of W concentration. This was mainly due to the oxidation of surface exposed $\mathrm{W}$, once the films are exposed to air after deposition. A. Schroeder et al. [24] suggested that once the films are exposed to air a gradual oxidation takes place more on the surface exposed metal particles in the first few days. Thereafter, no more significant additional change can be seen in the core level XPS spectra.

Fig. 1 shows the XPS wide survey spectra for both pure DLC and $\mathrm{W}-\mathrm{DLC}$ films with and without $\mathrm{Ar}^{+}$etching. The XPS survey spectrum of pure DLC film confirmed the existence of pure carbon bonding through the presence of sharp C1s peak. Similarly, the XPS survey spectrum of un-etched W-DLC film shows the presence of $\mathrm{C} 1 \mathrm{~s}, \mathrm{~W}$ $4 \mathrm{~s}, \mathrm{~W} 4 \mathrm{p}, \mathrm{W} 4 \mathrm{~d}$ and $\mathrm{O} 1 \mathrm{~s}$ bonding states. A sharp and more prominent $\mathrm{O}$ 1s peak confirms the atmospheric oxygen contamination on the surface exposed tungsten metals in DLC matrix. Hence in order to know more about the chemical bonding state of tungsten metal at the core, the as deposited W-DLC film was etched for about $4 \mathrm{~min}$ using high energy Argon ion sputtering. After $\mathrm{Ar}^{+}$etching for $4 \mathrm{~min}$, the XPS survey spectrum of W-DLC film confirms that there is no oxygen peak is present, only the metallic and carbon bonding states were observed. These results clearly confirm that the presence of pure carbon and tungsten phases at the core of W-DLC nanocomposite thin films.

Fig. 2 shows the W $4 \mathrm{f}$ XPS core level spectra for both un-etched and etched W-DLC3 (8.2 at.\% of W) nanocomposite thin films. W $4 \mathrm{f}$ spectra of un-etched thin film posses spin orbit doublet peaks, $W$ $4 f_{7 / 2}$ and $W 4 f_{5 / 2}$ with binding energy values of $35.5 \mathrm{eV}$ and $37.6 \mathrm{eV}$, respectively. These binding values match exactly with the tungsten oxide phase as reported by J.D. Reyes et al. [25]. Hence, in order to know the chemical reactivity between carbon and tungsten at the

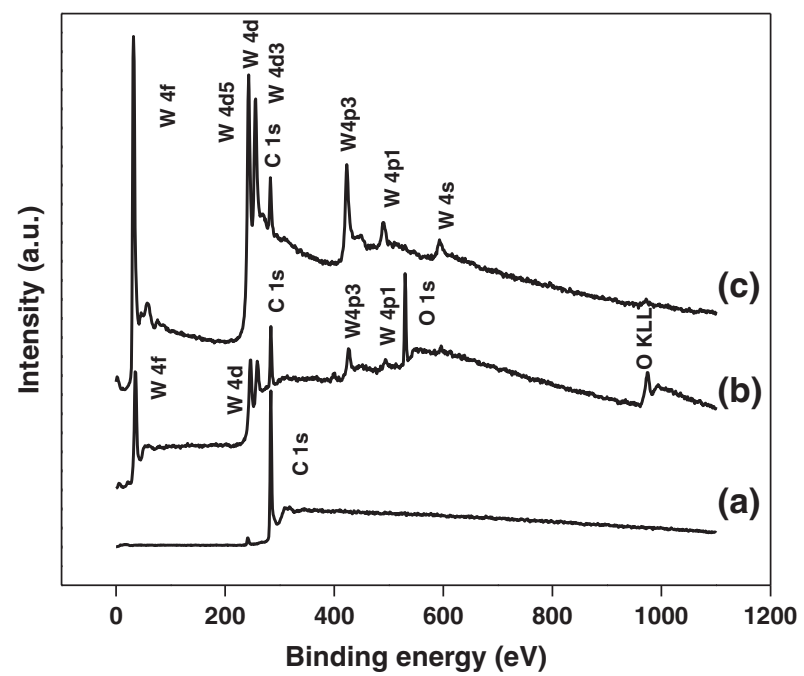

Fig. 1. XPS wide survey spectra of (a) pure DLC (b) un-etched W-DLC and (c) Etched W-DLC nanocomposite thin films.

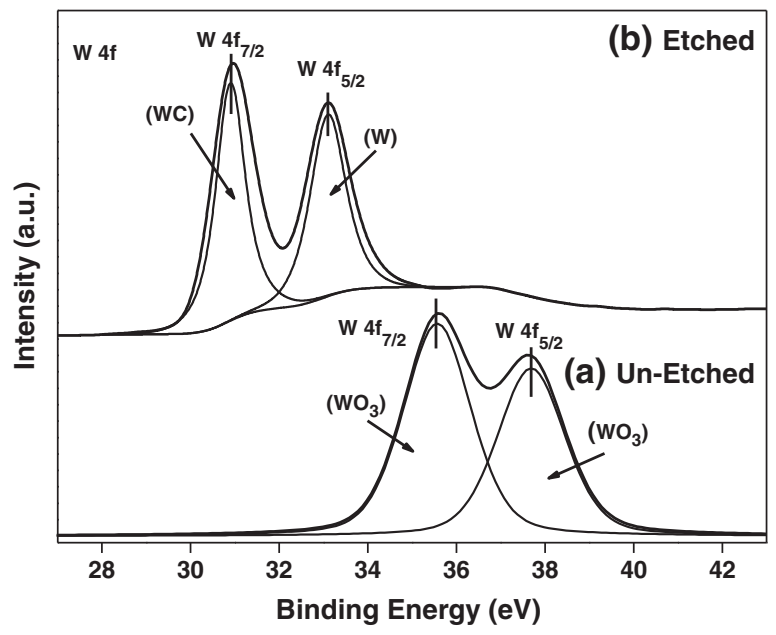

Fig. 2. Deconvoluted W $4 \mathrm{f}$ XPS spectra for W-DLC thin films (a) Un-etched W-DLC and (c) Etched W-DLC nanocomposite thin films.

core, the W-DLC3 sample surface was etched using $\mathrm{Ar}^{+}$ion beam for $4 \mathrm{~min}$. After argon etching, it shows doublet peaks at $30.8 \mathrm{eV}$ and $33.0 \mathrm{eV}$ corresponding to $\mathrm{WC}$ and pure metallic $\mathrm{W}$ phases respectively [26]. Thus from this etching analysis, it was clearly seen that at the core most of the incorporated $\mathrm{W}$ gets reacted with carbon to form tungsten carbide and some un-reacted tungsten nanoparticles were also present in W-DLC3 nanocomposite thin film. Moreover, there is no oxygen bonding is present at the core of these nanocomposite films. Hence this result additionally confirms the purity of the nanocomposite films and the deposition system. In general, most of the protective coating properties highly depend on the surface composition and chemical bonding. Thus in order to evaluate the naturally modified chemical bonding states on the surface of the W-DLC nanocomposite thin films, XPS analysis was further performed on airexposed (un-etched) W-DLC thin film samples.

Fig. 3 shows the influence of tungsten incorporation on the chemical bonding of un-etched DLC nanocomposite thin films. The $C 1 \mathrm{~s}$ and W $4 \mathrm{f}$ XPS spectra were deconvoluted by fixing the peak position and all the other parameters were left freely adjustable in XPSpeak 4.1 software. The incorporation of W into the DLC matrix, alters the $\mathrm{C} 1 \mathrm{~s}$ spectra and it composed with five different components with binding energies $\sim 283.3 \mathrm{eV}, \sim 283.7 \mathrm{eV}, \sim 284.5 \mathrm{eV}, \sim 285.2 \mathrm{eV}$ and $\sim 286.2 \mathrm{eV}$ correspond to $\mathrm{WC}, \mathrm{W}_{2} \mathrm{C}, \mathrm{C} \mathrm{sp} \mathrm{sp}^{2}, \mathrm{C} \mathrm{sp}$ and $\mathrm{C}-\mathrm{O}$ bonds, respectively $[27,28]$. From the deconvoluted $C$ 1s core level spectra, the relative proportion of $\mathrm{sp}^{3}$ and $\mathrm{sp}^{2}$ bonding states in the films were evaluated. The $\mathrm{sp}^{3} / \mathrm{sp}^{2}$ ratio decreases with increase in $\mathrm{W}$ content and which is 0.646 for $W$ free DLC and 0.17 for W-DLC5 film (W - 13.7 at.\%) which directly implies an increase in $\mathrm{sp}^{2}$ content. Thus increase in $\mathrm{sp}^{2}$ content with increase of $\mathrm{W}$ concentration in DLC matrix indicates that the tungsten favours the formation of graphitic $\left(\mathrm{sp}^{2}\right)$ bonds in DLC matrix. Similarly, the W 4f XPS core level spectra of un-etched W-DLC thin films were deconvoluted and it was found that most of the spectra were composed with two different binding energies around $\sim 35.8 \mathrm{eV}$ and $\sim 37.9 \mathrm{eV}$, which corresponds to $\mathrm{WO}$ and $\mathrm{WO}_{3}$ bonds, respectively $[27,29]$. However, the film with tungsten of 13.7 at.\%, posses four distinct features: They are $\sim 31.4 \mathrm{eV}$ and $\sim 33.5 \mathrm{eV}$ corresponding to pure $\mathrm{W}$ and other two binding energies at $\sim 35.5$ and $\sim 37.6 \mathrm{eV}$ correspond to tungsten oxides. It was clearly seen that in low tungsten incorporated DLC films, all the surface exposed tungsten nanoparticles were contaminated by oxygen to form tungsten oxide, but for high tungsten incorporated DLC film, along with the tungsten oxide phase pure metallic tungsten phases were observed. In addition to that of $\mathrm{W} 4 \mathrm{f}$ spectra, $\mathrm{O}$ 1s spectra (not shown here) also confirms that the surface of the nanocomposite thin films were contaminated by atmospheric oxygen and its concentration 
W-DLC1
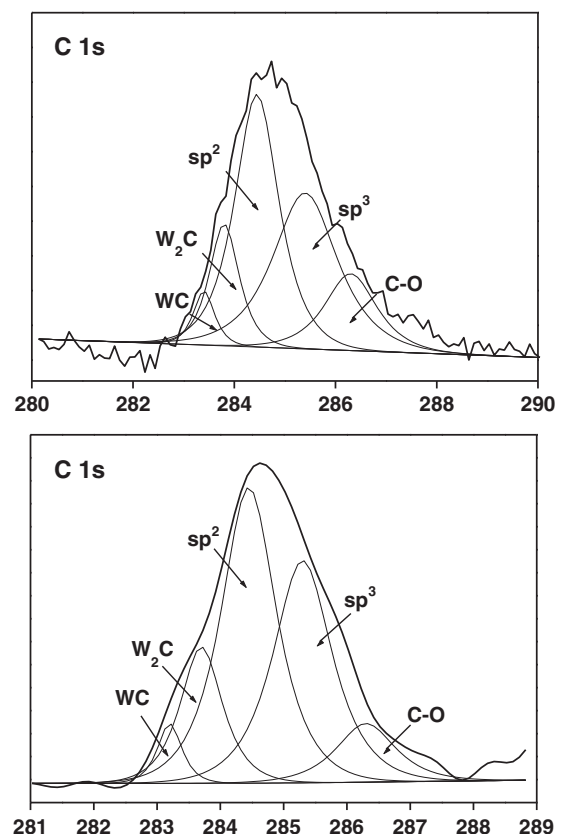

W-DLC2

W-DLC3

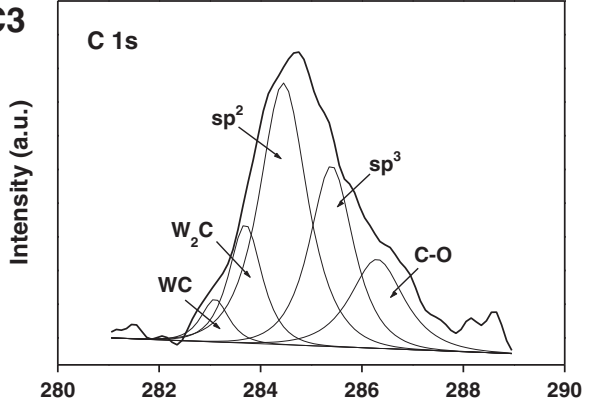

W-DLC4

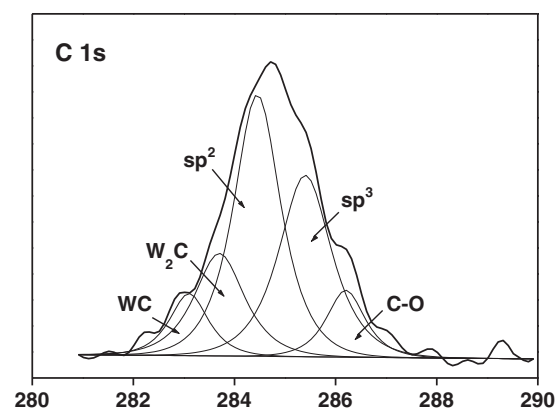

W-DLC5

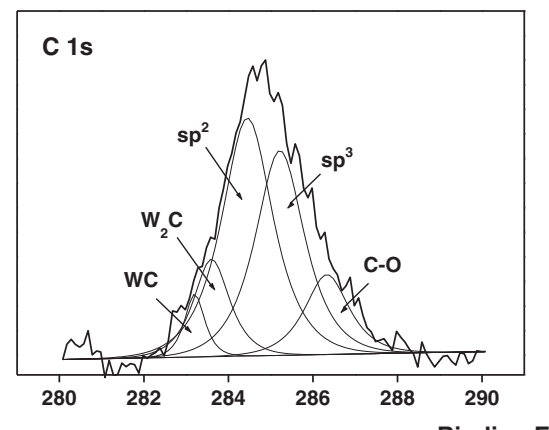

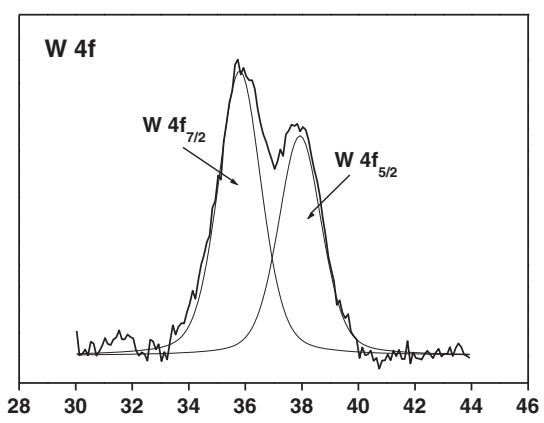

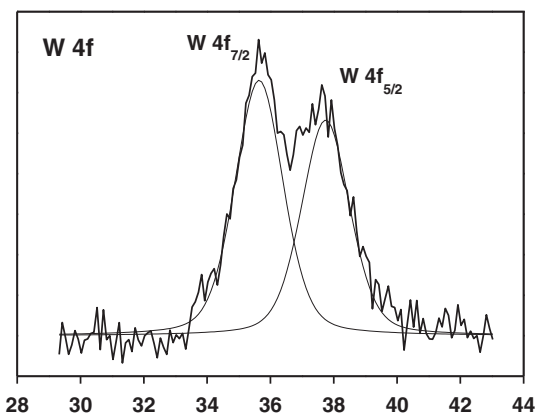

$\begin{array}{lllllllll}28 & 30 & 32 & 34 & 36 & 38 & 40 & 42 & 44\end{array}$
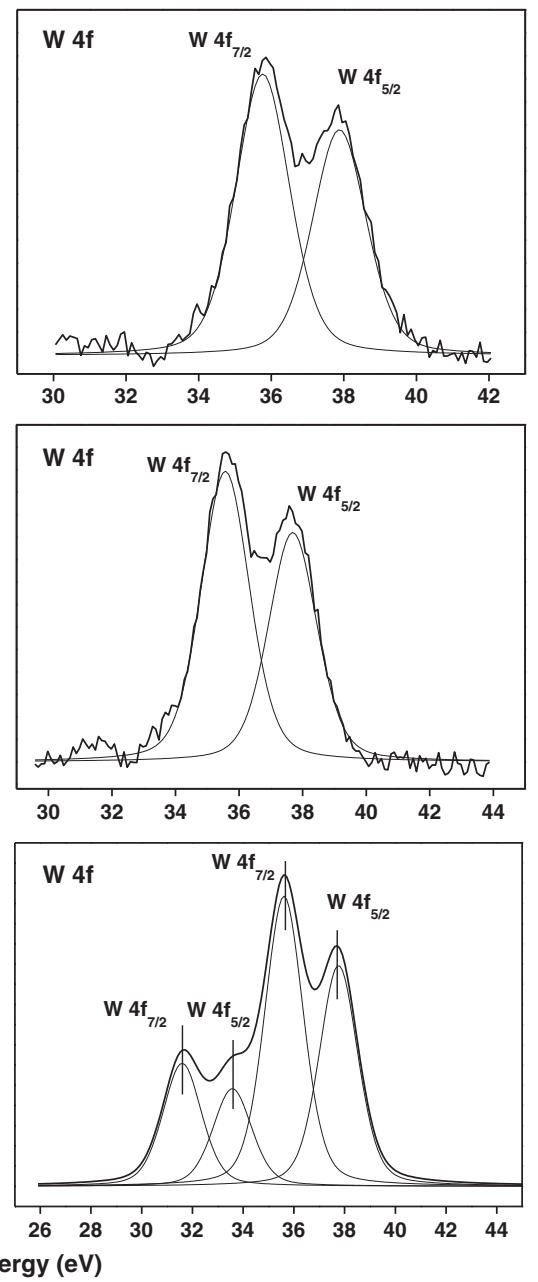

Fig. 3. Deconvoluted C1s and W 4f XPS spectra for un-etched W-DLC thin films with variable W fractions and marked as W-DLC1 to W-DLC5.

varies with respect to the presence of surface exposed tungsten metal in W-DLC thin films. All these observations suggest that the incorporated tungsten is bound in a carbidic form to the surrounding carbon atoms in the DLC matrix.

\subsection{Raman Spectroscopic Analysis}

Raman spectroscopy is a non-destructive and effective tool for the characterization of DLC films i.e. for the determination of the 
structural properties and composition of these films [30,31]. It is traditionally carried out at wavelengths in the visible spectral region, where the Raman scattering is much higher for $\mathrm{sp}^{2}$-bonded carbon than for the $\mathrm{sp}^{3}$-bonded one, the Raman spectrum is dominated by spectral features connected with the $\mathrm{sp}^{2}$-bonded phase. However, the information regarding the $\mathrm{sp}^{3}$-bonded phase can be indirectly obtained by the direct linkage between $\mathrm{sp}^{2}$ and $\mathrm{sp}^{3}$ phases. Usually, all carbons show their characteristic features in their Raman spectra in $800-2000 \mathrm{~cm}^{-1}$ region, the so called G and D peaks, which lies at around 1560 and $1360 \mathrm{~cm}^{-1}$, for visible exicitation [31,32]. The G peak is due to the bond stretching of all pairs of $\mathrm{sp}^{2}$ atoms in both rings and chains, and the $\mathrm{D}$ peak is due to the breathing modes of $\mathrm{sp}^{2}$ atoms in rings. Many previous studies have shown that the $\mathrm{sp}^{3}$ fraction of the films can be calculated from the wave number and the intensity of the $G$ and $D$ peaks. As the $\mathrm{sp}^{3}$ fraction decreases, the $\mathrm{G}$ peak shifts towards higher wavenumber and the ratio of $I(\mathrm{D}) / \mathrm{I}(\mathrm{G})$ increases [30-33]. Fig. 4 shows the Raman spectra for (a) pure DLC and (b) W-DLC films with different W fractions (6.1 to 13.7 at.\%). The Raman spectra were deconvoluted using Gaussian fitting into two main Raman peaks as the D peak and the G peak. Additionally a broad peak around $960 \mathrm{~cm}^{-1}$ corresponds to the second-order peak of the Si substrate which confirms that the films are of low thickness and transparent to visible Raman laser beam. Fig. 5 shows the corresponding $I(D) / I(G)$ ratio and the $G$ peak position of the pure DLC film and W-DLC nanocomposite films. It was clearly seen that the $G$ peak position shifts towards higher wavenumber and the $I(D) / I(G)$ ratio gets increased when the $\mathrm{W}$ concentration increased from 0 to 13.7 at.\%. This result clearly confirms that the incorporation of tungsten could favour more for the formation of $\mathrm{sp}^{2}$ bonded carbon sites in the amorphous DLC matrix. Recently, few researchers $[10,34,35]$ have assumed that in metal incorporated DLC nanocomposite films, the presence of small sized amorphous metal domains can act effectively to promote the formation of $\mathrm{sp}^{2}$ sites. Also with the increase in the metal concentration, more metal atoms would preferentially get reacted and bonded with $\mathrm{sp}^{2}$ carbon to form the metal carbide phase, owing to the relative lower bond energy compared with $\mathrm{sp}^{3}$ $\mathrm{C}$ sites. The present research also gives evidence to this supposition that on increasing the $\mathrm{W}$ concentration, excess formation of tungsten carbide phase which in turn increases the $\mathrm{sp}^{2}$-C sites in the DLC host matrix. Also the intensity of the spectra decreased markedly, because of the presence and increasing fraction of Raman inactive WC phase at the surface/core of the amorphous DLC film. From the obtained XPS results also it was clearly proved that with the increase in the

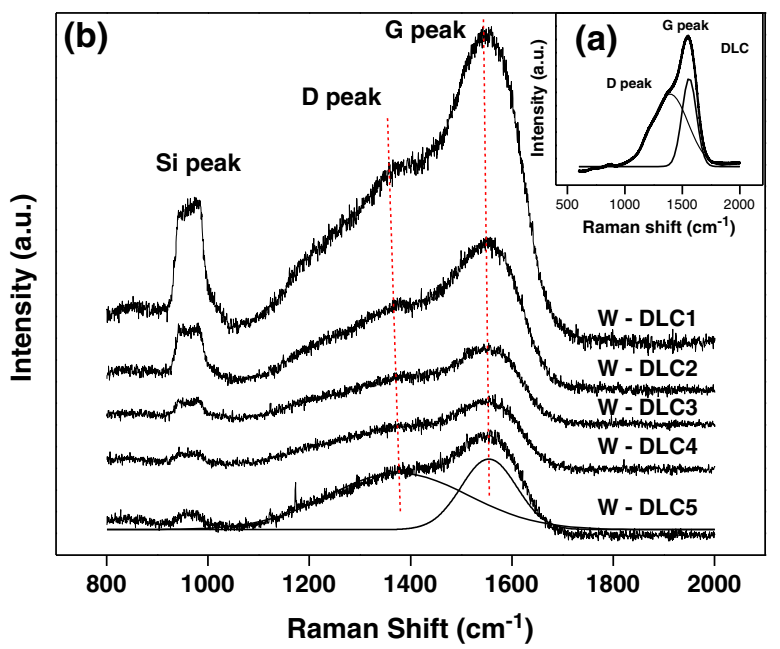

Fig. 4. Raman spectra for (a) pure DLC and (b) W-DLC nanocomposite thin films with different $\mathrm{W}$ content, dashed line marks the corresponding D peak and $\mathrm{G}$ peak.

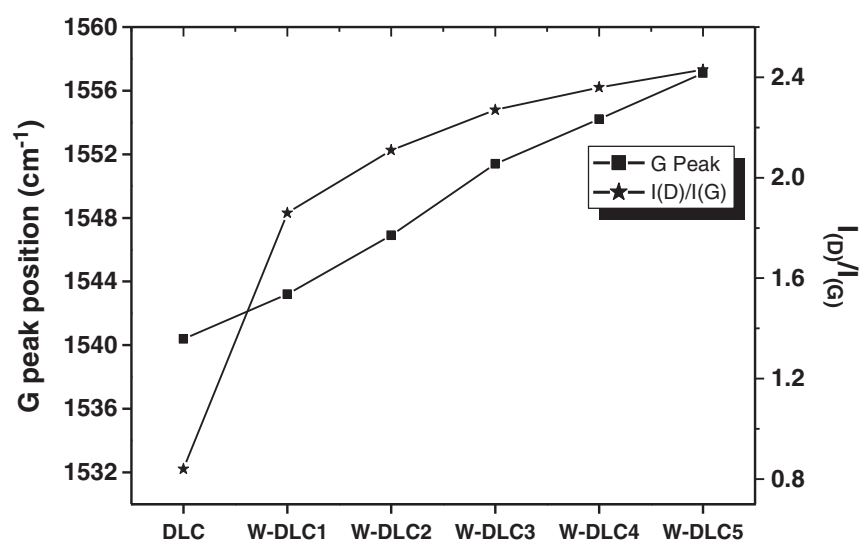

Fig. 5. Evolution of ID/IG ratio and $G$ peak position with respect to variation in the $W$ concentration in W-DLC nanocomposite thin film samples.

concentration of $\mathrm{W}$ to 13.7 at.\%, the $\mathrm{W} 4 \mathrm{f}$ core level spectra shows doublet peaks corresponding to the presence of excess WC phase along with the weakly bonded $\mathrm{WO}_{3}$ at the surface of the nanocomposite thin films.

\subsection{Transmission Electron Microscopic Analysis (TEM)}

Fig. 6(a-c) shows plan-view TEM image, high resolution TEM image and Fast Fourier transform (FFT) pattern of W-DLC5 (W 13.7 at.\%) nanocomposite thin film. The TEM image of as prepared
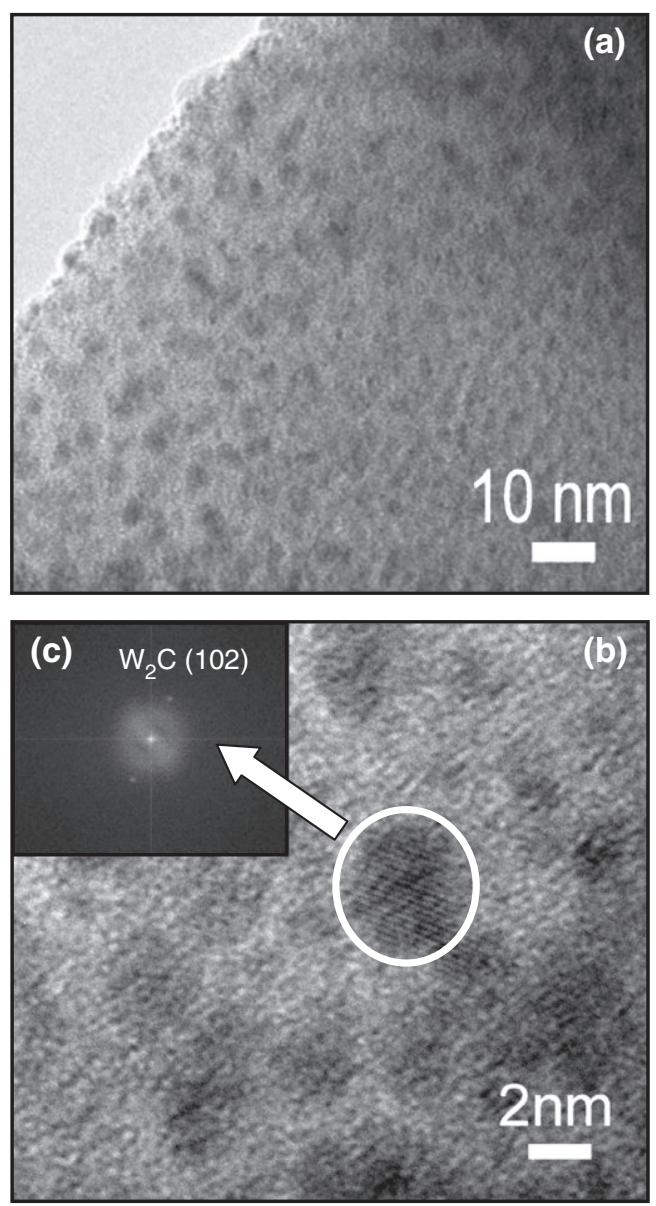

Fig. 6. TEM image of W-DLC thin film (a) plan view TEM image (b) HRTEM image and (c) FFT pattern of W-DLC5 (W - 13.7 at.\%) nanocomposite thin film. 
W-DLC5 sample reveals that the incorporated $\mathrm{W}$ atoms reacted with carbon to form spherical shaped tungsten carbide $\left(\mathrm{W}_{2} \mathrm{C}\right)$ clusters. From the HRTEM image (Fig. $6(\mathrm{~b})$ ) it was clearly seen that the $\mathrm{W}_{2} \mathrm{C}$ clusters are of $\sim 3 \mathrm{~nm}$ in size and were uniformly dispersed in the amorphous DLC host matrix. In addition to that the FFT pattern (Fig. 6 (c)) reveals that the $\mathrm{W}_{2} \mathrm{C}$ nanoclusters exist in the hexagonal (a)

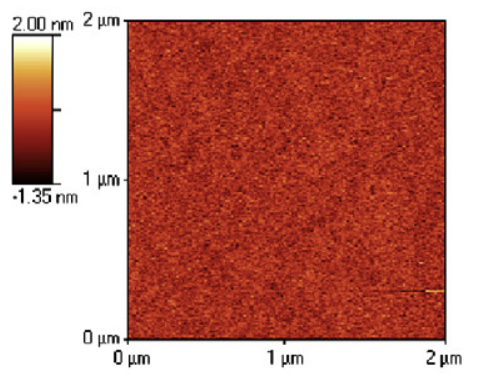

(d)

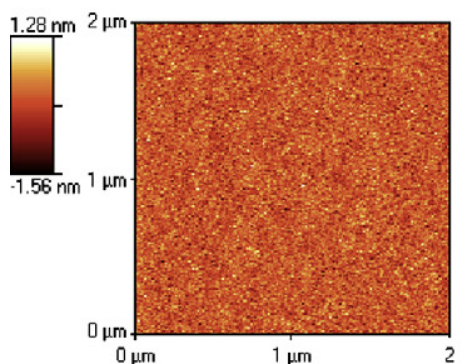

DLC

(b)

(c)
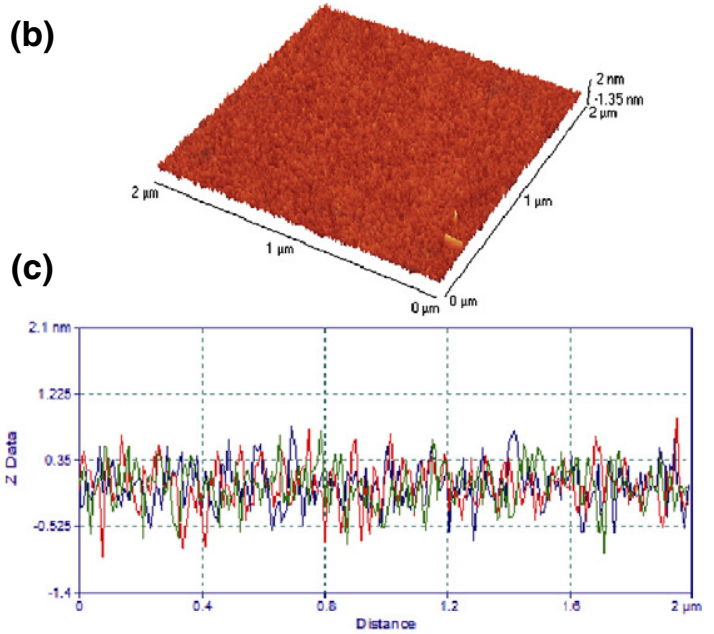

W-DLC1

(e)

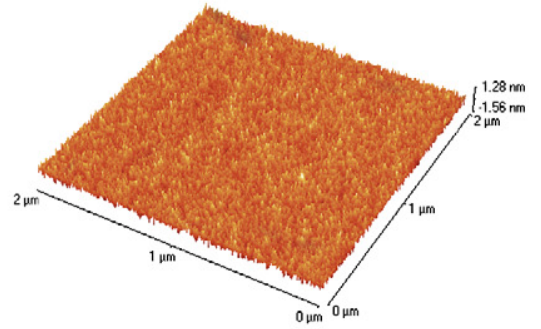

(f)

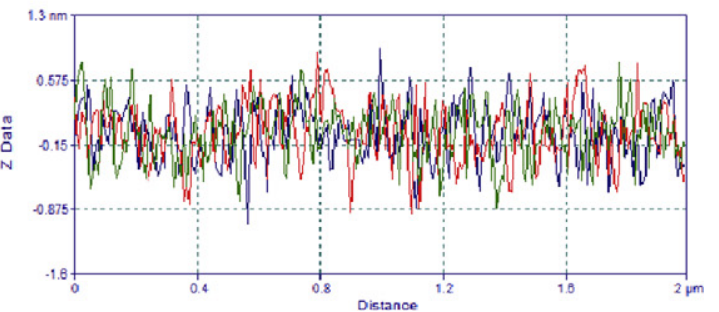

(g)

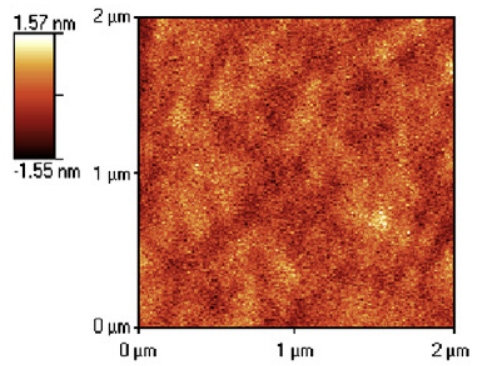

W-DLC5

(h)

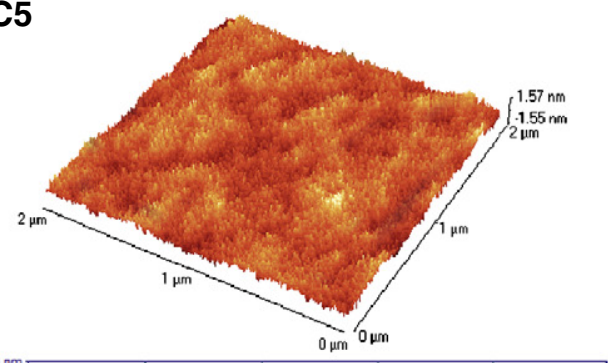

(i)

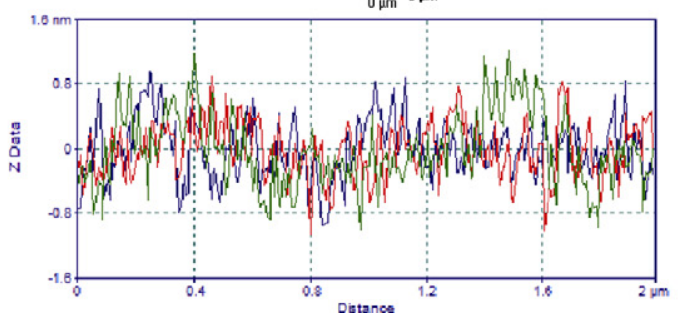

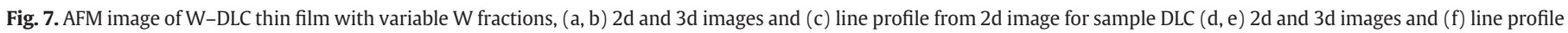
from 2d image for sample W-DLC1 and $(\mathrm{g}, \mathrm{h})$ 2d and 3d images and (i) line profile from 2d image for sample W-DLC5. 
close packed crystal structure with crystallographic orientation of (102). Recently it was reported that the size and the distribution of the tungsten carbide cluster mainly depend on $\mathrm{W}$ concentration $[10,13,36,37]$. In the present research also we observed complete dissolution of incorporated $\mathrm{W}$ atoms in the amorphous DLC matrix, at very low $\mathrm{W}$ fractions. But by increasing the $\mathrm{W}$ concentration to 13.7 at.\%, the incorporated $\mathrm{W}$ reacts with carbon to form crystalline WC nanoclusters and gets dispersed well in the amorphous DLC network. This may be due to the segregation of amorphous WC phase at this concentration and eventually get evolved into a nano-sized crystalline $\alpha-\mathrm{W}_{2} \mathrm{C}$ phase. XPS and Raman spectra results support this assumption by confirming the presence and the formation of WC phase with increasing the concentrations of W in W-DLC nanocomposite thin films. Especially Raman spectra of W-DLC films confirms by decreasing the intensity of the spectra with the increase in the segregation of WC fractions in the DLC matrix.

\subsection{Atomic Force Microscopic Analysis (AFM)}

Fig. 7(a-i) shows the surface topography of $\mathrm{W}$ incorporated DLC films deposited with different $\mathrm{W}$ fractions ( 0 to 13.7 at.\%). These images give a clear understanding about the topographical changes in the composite coatings. These films are uniform and highly smooth, and are composed of small column like structures. The size of the column like structures gets increased with increasing $\mathrm{W}$ concentration. The formation of these columns like structure may be due to the delocalizing of $\mathrm{sp}^{2}$ sites after $\mathrm{W}$ incorporation [38]. From the surface topography images, the surface roughness (rms) of DLC film is found to be $0.25 \mathrm{~nm}$, whereas with the increase in $\mathrm{W}$ concentration from 6.2 at.\% to 13.71 at.\% in the DLC matrix, the surface roughness gets increased from 0.28 to $0.38 \mathrm{~nm}$. In addition to surface roughness and surface topographic analysis, the line profile analysis was performed to retrieve the detailed information about the surface uniformity of the W-DLC nanocomposite thin films. Three lines are randomly chosen in three different places on the surface of the film to analyze their uniformity. The line profile analysis also confirms that the incorporation of $\mathrm{W}$ into the DLC matrix has increased the peak-to valley distance from 0.6 to $3.6 \mathrm{~nm}$ and the obliquity of the slope shifts from 2 to $4^{\circ}$. The line profile analysis complements the surface roughness values. This could be due to the growth of spherical shaped nanocluster structures with the increase in the $\mathrm{W}$ concentrations.

\subsection{Mechanical properties}

The effect of tungsten incorporation on the mechanical properties of $\sim 130 \mathrm{~nm}$ W-DLC nanocomposite thin films was determined using depth sensitive nanoindentation technique. At an indentation depth around $26 \mathrm{~nm}$ (approximately 30\% of the film thickness), the hardness, $H$ value is $25.6 \mathrm{GPa}$ and $18.0 \mathrm{GPa}$ for the DLC film and W-DLC5 sample ( $\mathrm{W}-13.7$ at.\%), respectively. It can be seen from Fig. 8(a) that the $H$ of films decreases with the increase in indentation depth, indicating that the measured $H$ value is not the hardness value of the only film but a combination of both the film and the substrate. The effect of substrate increased with increasing the indenter penetration depth and $H$ values reaches to $\sim 12 \mathrm{GPa}$ for Si. However at the initial depth (i.e. $<30 \mathrm{~nm}$ ), it can be clearly seen that the $H$ of the films decreases with the increase of W concentration in the DLC films. The incorporation of tungsten into DLC matrix diminishes the ability to resist plastic deformation because of breakage of $\mathrm{C}-\mathrm{C}$ bonds and formation of $\mathrm{W}-\mathrm{C}$ bonds. Upto a concentration of 10.4 at.\% of $\mathrm{W}$, the variation in the hardness was small but drastic decrease was observed at 13.7 at.\%. Nevertheless, Wang et al. [13] have reported that the changes in the mechanical properties were not significant with increasing $\mathrm{W}$ at.\%. In contrast, we have observed a gradual decrease in hardness with increasing $\mathrm{W}$ concentration. This may be due to the increase of $\mathrm{C}-\mathrm{sp}^{2}$ content and the well isolation of (a)
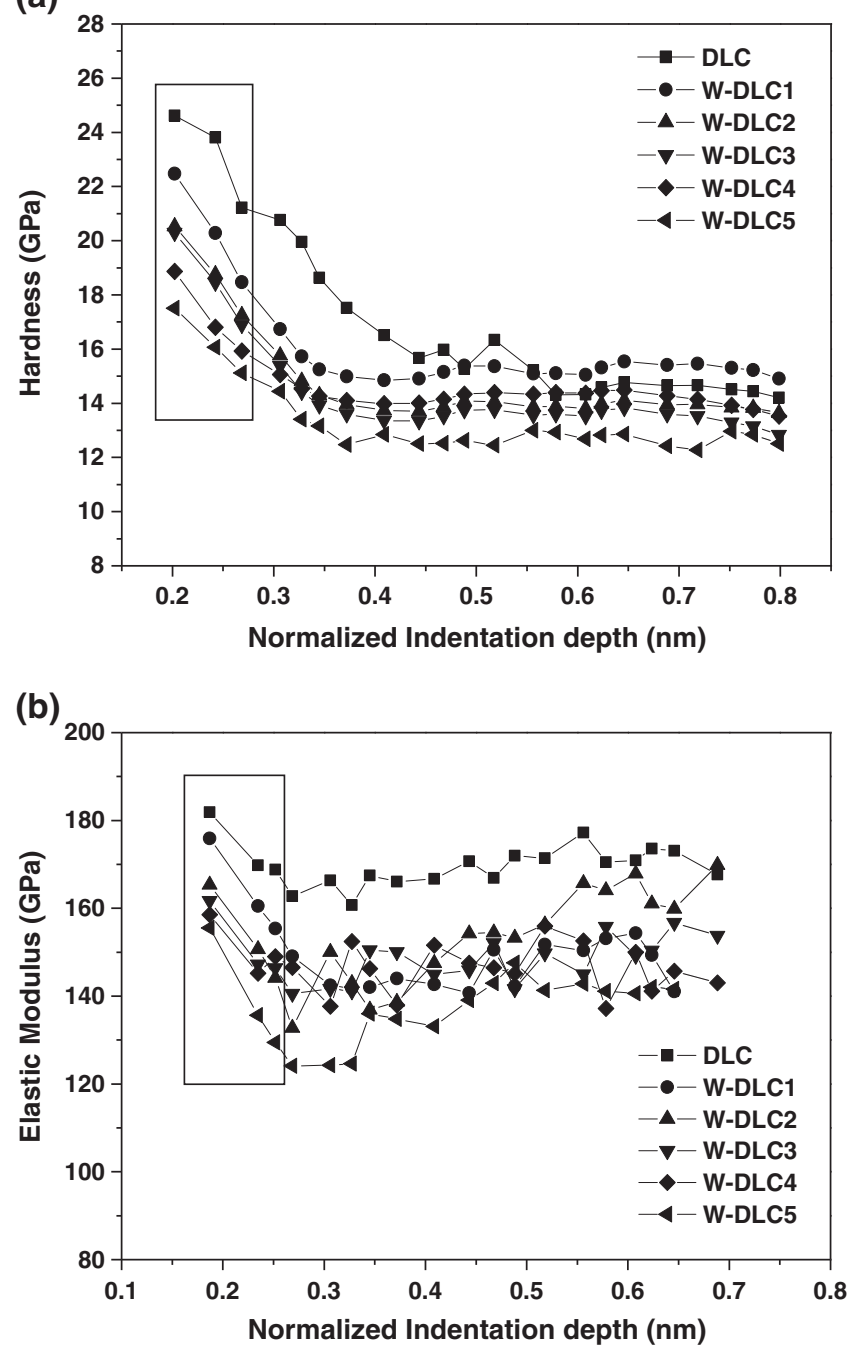

Fig. 8. (a): Variations of film hardness as a function of indenter penetration depth for pure DLC films and W-DLC films with different tungsten fractions.(b): Variation of elastic modulus with varying tungsten fractions in the DLC films.

carbide particle in the amorphous carbon matrix as confirmed by XPS and TEM results discussed earlier. Additionally, the obtained mechanical hardness is much higher than the hardness observed for $\mathrm{H}$ containing and $\mathrm{H}$-free W-DLC films prepared by Silva et al. using reactive RF sputtering [39] and our early work on W based carbon films [21]. Elastic modulus, $E$, which is a measure of stiffness, followed the same trend as that of hardness. The $E$ of pure DLC film is around $\sim 180 \mathrm{GPa}$. With the incorporation of W into the DLC matrix, $E$ gradually decreased to 160 GPa (shown in Fig. 8(b)). However, the film with 13.7 at.\% of W has exhibited lower value of $E$ to $\sim 140 \mathrm{GPa}$. The decrease in $E$ with increase in W at.\% is due to the higher bond length of $\mathrm{W}-\mathrm{C}$ than $\mathrm{C}-\mathrm{C}$ and this would play a significant role in the range of composition studied. Overall, it can be concluded from the obtained results that though the incorporation of tungsten induce more growth of $\mathrm{C}-\mathrm{sp}^{2}$ bonding sites in the DLC matrix, the presence of hard WC nanoclusters and uniform distribution of nanoclusters in the amorphous carbon matrix helps to restraining the loss of hardness and elastic modulus of W-DLC nanocomposite thin films.

The effect of $\mathrm{W}$ incorporation on the behaviour of DLC films was investigated using the pile-up formation after indentation. For this, the surface topographic images of DLC and W-DLC films were captured immediately after indentation on the indented zone using insitu piezo-electric scanner. The SPM topographical images of pure 

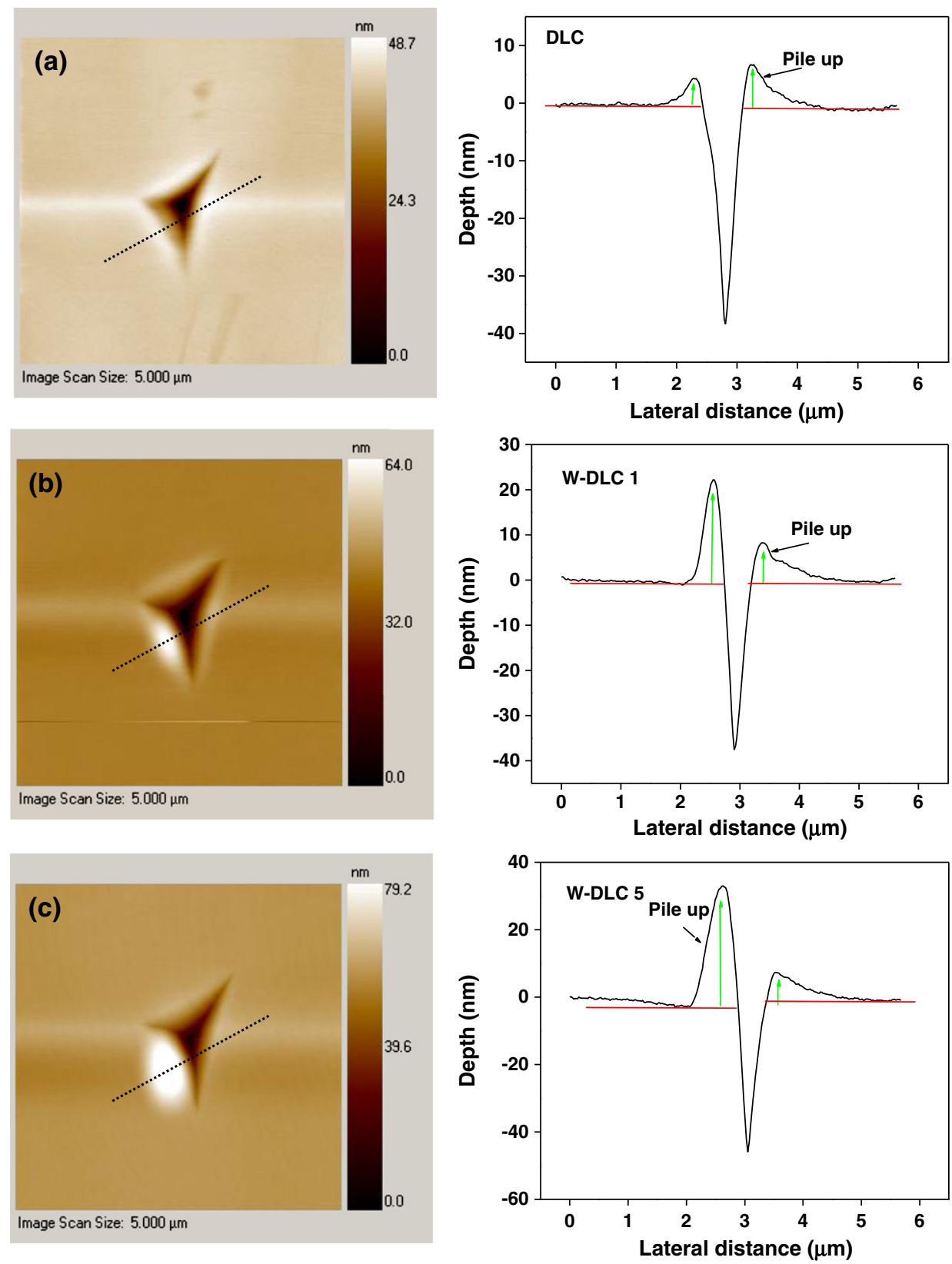

Fig. 9. Surface topographies and their corresponding line profile on the residual indent impression of (a) DLC (b) W-DLC1 and (c) W-DLC5 thin films.

DLC, W-DLC1 and W-DLC5 film surfaces are shown in Fig. 9(a-c). The magnitude of pile-up was measured from the difference in the peak to valley as marked in Fig. 9(a-c). Interestingly, no cracking or chipping was observed and only some pile-up was observed on the side of the residual indent from the W-DLC thin films, as shown in Fig. 9(b and c). This equal amount of pile-up of material on either side is due to the isotropic nature of the film. However, a similar observation on pure DLC films revealed slight pile-up formation, as shown in Fig. 9(a). Usually, depending upon the degree of strain hardening of the tested material, the surface deformation mode of an indent can have pile-up or sink-in. Therefore, it is clear from the residual indent images that $\mathrm{W}$ incorporation induces more incompressible plastic deformation in the DLC films during the indentation.

\subsection{Nanoscratch-adhesion analysis}

The friction coefficient and adhesion behaviour of pure DLC film was compared with W-DLC nanocomposite thin film using nanoscratch test under ramping load from 0 to $1 \mathrm{mN}$. Fig. 10 shows the friction coefficient as a function of lateral displacement from 0 to $10 \mu \mathrm{m}$. In general, while comparing the friction coefficient values of both the pure DLC and W-DLC films it was found that they posses low friction coefficient values and highly scratch resistance without any peel off from the substrate under higher scratch loads. The typical value of friction coefficient is 0.19 for pure DLC, 0.11 for W-DLC1 and 0.03 for W-DLC5. These coefficients have been evaluated as the average value of friction coefficient along a scratch track. It was clearly seen that the friction coefficient decreases with the increase in the $\mathrm{W}$ concentration in these nanocomposite thin films. Both the DLC and W-DLC nanocomposite thin films show superior scratch resistance and does not undergo any peel off from the substrate upto a maximum load of $1 \mathrm{mN}$. The existence of spherical shaped hard WC nanoclusters in the amorphous DLC matrix hinders the stress propagation which in turn increased the critical load bearing capacity of W-DLC compared to that of pure DLC thin film. In addition to that the surface topography of the scratch track on the thin films was monitored using the AFM analysis (shown in Fig. 11(a and b)). It was 


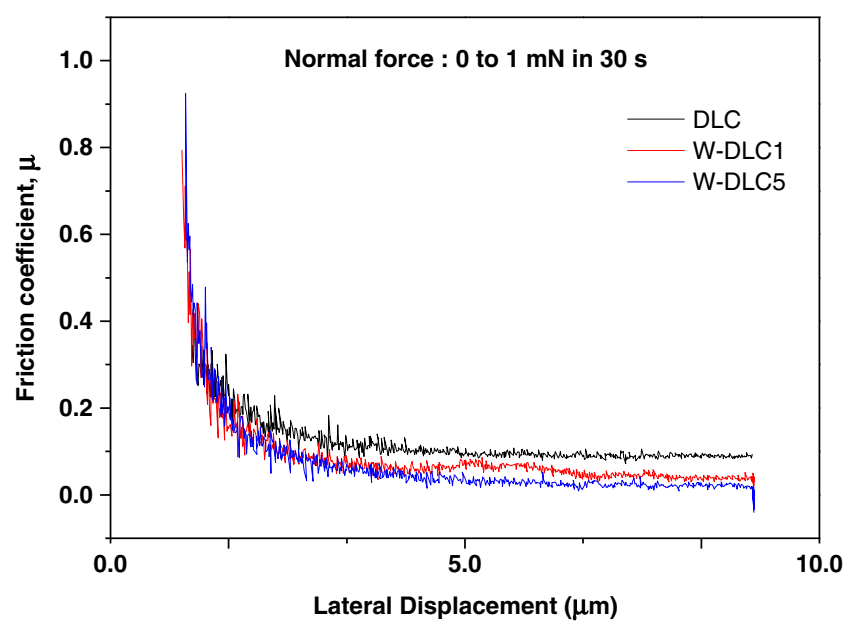

Fig. 10. Variations of friction coefficient as a function of tungsten concentration in W-DLC thin with a ramping load from 0 to $1 \mathrm{mN}$ in $30 \mathrm{~s}$.

clearly seen that there is thin scratch track and no peel off was observed for both DLC and W-DLC nanocomposite thin films. However, the depth impression due to the scratch was found to be twice deep for pure DLC compared to that of W-DLC5 nanocomposite thin films. Hence it can be concluded from the scratch test results that the W-DLC films prepared through RBTIBD method are high smooth and highly scratch resistant within this load range.

\section{Conclusions}

Tungsten incorporated DLC nanocomposite films with variable fractions of $\mathrm{W}$ ranging from 0 to 13.7 at.\% was successfully prepared by using reactive-biased target ion beam deposition technique. It was observed that $\mathrm{W}$ concentration in the films increased with an increasing target bias voltage. It was found that by increasing the $\mathrm{W}$ fractions, most of the incorporated $\mathrm{W}$ reacts with carbon to form
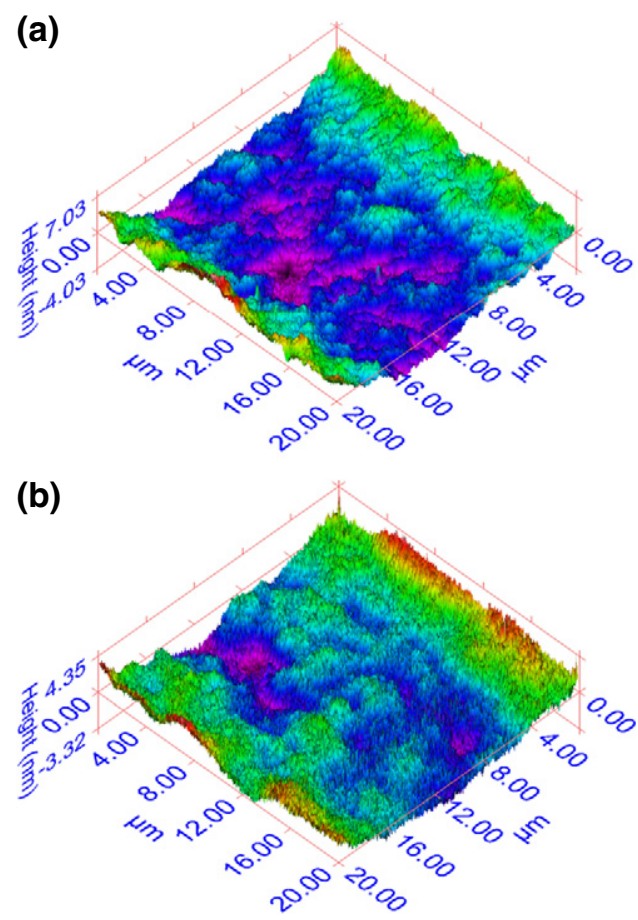

Fig. 11. The surface topographies of (a) DLC and (b) W-DLC5 thin film samples after the scratch test.
WC nanoclusters and was dispersed uniformly throughout the DLC matrix. Also the $\mathrm{C}-\mathrm{sp}^{2}$ hybridised bonding in the DLC host matrix gets increased with increasing $\mathrm{W}$ fraction. Moreover, the homogenous distribution of $3 \mathrm{~nm}$ spherical shaped WC nanoclusters favoured for restraining the loss of mechanical hardness and elastic modulus of W-DLC films. Additionally, nano-scratch test confirmed that the presence of unique nanocomposite structure favoured the W-DLC for very low friction coefficient compared to that of pure DLC and promising scratch resistance. Among these films, the one with low $\mathrm{W}$ fraction exhibit excellent mechanical hardness along with low friction coefficient. The results obtained thus suggest that through the present deposition technique it is possible to prepare smooth and thin tungsten based carbon nanocomposite thin film with uniform distribution of metal carbide nanoclusters with excellent mechanical and frictional properties suitable for thin protective coating applications.

\section{Acknowledgment}

One of the authors, PVB, would like to thank the Government of Canada, International Scholarship Programs for providing partial financial support to carry out this research work in University of Saskatchewan, Canada. Authors also thank Dr. J.Silvestre-Albero, Department of Inorganic Chemistry, University of Alicante, Spain for helping in XPS analysis.

\section{References}

[1] J. Robertson, Mater. Sci. Eng. R 37 (2002) 129-281.

[2] Geoffrey Deamaley, James H. Arps, Surf. Coat. Technol. 200 (2005) 2518-2524.

[3] Pi-Chuen Tsai, Yen-Fei Hwang, Jueh-Yu Chiang, Wen-Jauh Chen, Surf. Coat. Technol. 202 (2008) 5350-5355.

[4] Yin-Yu Chang, Da-Yung Wang, WeiTe Wu, Thin Solid Films 420-421 (2002) 241-247.

[5] Fei Zhao, Hongxuan Li, Li Ji, Yongjun Wang, Huidi Zhou, Jianmin Chen, Diamond Relat. Mater. 19 (2010) 342-349.

[6] R.D. Mansano, R. Ruas, A.P. Mousinho, L.S. Zambom, T.J.A. Pinto, L.H. Amoedo, M. Massi, Surf. Coat. Technol. 202 (2008) 2813-2816.

[7] N. Benchikh, F. Garrelie, C. Donnet, K. Wolski, R.Y. Fillit, F. Rogemond, J.L. Subtil J.N. Rouzaud, J.Y. Laval, Surf. Coat. Technol. 200 (2006) 6272-6278.

[8] D. Caschera, F. Federici, S. Kaciulis, L. Pandolfi, A. Cusma, G. Padeletti, Mater. Sci. Eng.C 27 (2007) 1328-1330.

[9] Koumei Baba, Ruriko Hatada, Surf. Coat. Technol. 169-170 (2003) 287-290.

[10] C. Corbella, E. Bertran, M.C. Polo, E. Pascual, J.L. Andujar, Diamond Relat. Mater. 16 (2007) 1828-1834

[11] Li Ji, Hongxuan Li, Fei Zhao, Jianmin Chen, Huidi Zhou, Diamond Relat. Mater. 17 (2008) 1949-1954

[12] K. Sedlackova, Zs. Czigany, T. Ujvari, I. Bertoti, R. Grasin, Gy.J. Kovacs, G. Radnoczi Nanotechnology 18 (2007) 445604.

[13] Ai-Ying Wang, Kwang-Ryeol Lee, Jae-Pyoung Ahn, Jun Hee Han, Carbon 44 (2006) 1826-1832.

[14] Y.M. Foong, A.T.T. Koh, H.Y. Ng, D.H.C. Chua, J. Appl. Phys. 110 (2011) 054904.

[15] Ming Xu, Wei Zhang, Zhengwei Wu, Shihao Pu, Liuhe Li, Paul K. Chu, J. Appl. Phys. 102 (2007) 113517.

[16] Wan-Yu Wu, Jyh-Ming Ting, Carbon 44 (2006) 1210-1217.

[17] Y.M. Foong, J. Hsieh, X. Li, D.H.C. Chua, J. Appl. Phys. 106 (2009) 064904.

[18] V.V. Uglov, A.K. Kuleshov, M.V. Astashynskaya, V.M. Anishchik, S.N. Dub, F. Thiery, Y. Pauleau, Compos. Sci. Technol. 65 (2005) 785-791.

[19] Wei Dai, Aiying Wang, Surf. Coat. Technol. 205 (2011) 2882-2886.

[20] Y. Tang, Y.S. Li, Q. Yang, A. Hirose, Appl. Surf. Sci. 257 (2011) 4699-4705.

[21] P. Vijai Bharathy, D. Nataraj, D. Mangalaraj, M.S.R.N. Kiran, J. Silvestre-Albero, Q Yang, Int. J. NanoScience. 10 (2011) 851-855.

[22] P. Vijai Bharathy, D. Nataraj, Paul K. Chu, Huaiyu Wang, Q. Yang, M.S.R.N. Kiran, J Silvestre-Albero, D. Mangalaraj, Appl. Surf. Sci. 257 (2010) 143-150.

[23] W.C. Oliver, G.M. Pharr, J. Mater. Res. 19 (2004) 3-20.

[24] Anouk Schroeder, Gilbert Francz, Arend Bruinink, Roland Hauert, Joerg Mayer Erich Wintermantel, Biomaterials 21 (2000) 449-456.

[25] J.D. Reyes, R.J. Delgado-Macuil, V. Dorantes-Garcia, A. Perez-Benitex, J.A. BalderasLopez, J.A. Ariza-Ortega, Mater. Sci. Eng. B 174 (2010) 182-186.

[26] J. Luthin, Ch. Linsmeier, Surf. Sci. 454-456 (2000) 78-82.

[27] J.F. Moulder, W.F. Stickle, P.E. Sobol, K.D. Bomben, in: J. Chastain (Ed.), Physical Electronic Division, Eden Prairie, MN, 2nd edn., Perkin-Elmer Corp., 1992

[28] Takanori Takeno, Takao Komiyama, Hiroyuki Miki, Toshiyuki Takagi, Takashi Aoyama, Thin Solid Films 517 (2009) 5010-5013.

[29] Othon R. Monteiro, Marie-Paule Delplancke-Ogletree, G. Ian Brown, Thin Solid Films 342 (1999) 100-107.

[30] C. Casiraghi, A.C. Ferrari, J. Robertson, Phys. Rev. B 72 (2005) 085401-085415.

[31] A.C. Ferrari, J. Robertson, Phys. Rev. B 61 (2000) 14095-14107.

[32] G. Irmer, A. Dorner-Reisel, Adv. Eng. Mater. 7 (2005) 694-705. 
[33] A.C. Ferrari, J. Robertson, Phys. Rev. B 64 (2001) 075414.

[34] N.W. Khun, E. Liu, G.C. Yang, Surf. Coat. Technol. 204 (2010) 3125-3130.

[35] Heon Woong Choi, Reinhold H. Dauskardt, Seung-Cheol Lee, Kwang-Ryeol Lee, Kyu Hwan Oh, Diamond Relat. Mater. 17 (2008) 252-257.

[36] C. Strondl, N.M. Carvalho, J.T.H.M. De Hosson, G.J. van der Kolk, Surf. Coat. Technol. 162 (2003) 288.
[37] Xinchun Chen, Zhijian Peng, Zhiqiang Fu, Sudong Wu, Wen Yue, Chengbiao Wang, Surf. Coat. Technol. 205 (2011) 3631-3638.

[38] E. Liu, X. Shi, B.K. Tay, L.K. Cheah, H.S. Tan, J.R. Shi, J. Appl. Phys. 86 (1999) 6078-6084.

[39] Carlos W. Moura e Silva, Jose R.T. Branco, Albano Cavaleiro, Solid State Sci. 11 (2009) 1778-1782. 